\title{
Community health workers improve diabetes care in remote Australian Indigenous communities: results of a pragmatic cluster randomized controlled trial
}

Robyn A McDermott ${ }^{1,2^{*}}$, Barbara Schmidt ${ }^{1}$, Cilla Preece ${ }^{1}$, Vickie Owens ${ }^{1}$, Sean Taylor ${ }^{1,2}$, Ming Li ${ }^{1}$ and Adrian Esterman ${ }^{1}$

\begin{abstract}
Background: Health outcomes for Indigenous Australians with diabetes in remote areas remain poor, including high rates of avoidable complications which could be reduced with better primary level care. We aimed to evaluate the effectiveness of a community-based health-worker led case management approach to the care of Indigenous adults with poorly controlled type 2 diabetes in primary care services in remote northern Australia.

Methods: Two hundred and thirteen adults with poorly controlled diabetes (HbA1c > 8.5\%) and significant comorbidities in 12 remote communities were randomly assigned by service cluster to receive chronic care co-ordination from a community-based health worker supported by a clinical outreach team, or to a waitlist control group which received usual care.

Results: At baseline, mean age of participants was 47.9 years, $62.4 \%$ were female, half were Aboriginal and half identified as Torres Strait Islander, 67\% had less than 12 years of education, 39\% were smokers, median income was $\$ 18,200$ and $47 \%$ were unemployed. Mean HbA1c was $10.7 \%(93 \mathrm{mmol} / \mathrm{mol})$ and BMI 32.5. At follow-up after 18 months, $\mathrm{HbA} 1 \mathrm{c}$ reduction was significantly greater in the intervention group $(-1.0 \% \mathrm{vs}-0.2 \%$, SE $($ diff $)=0.2$, $p=0.02$ ). There were no significant differences between the groups for blood pressure, lipid profile, BMl or renal function. Intervention group participants were more likely to receive nutrition and dental services according to scheduled care plans. Smoking rates were unchanged.

Conclusions: A culturally safe, community level health-worker led model of diabetes care for high risk patients can be effective in improving diabetes control in remote Indigenous Australian communities where there is poor access to mainstream services. This approach can be effective in other remote settings, but requires longer term evaluation to capture accrued benefits.
\end{abstract}

Trial registration: ANZCTR 12610000812099, Registered 29 September 2010.

Keywords: Type 2 diabetes, Australian aboriginal adults, Primary health care, Indigenous health workers

\footnotetext{
* Correspondence: robyn.mcdermott@jcu.edu.au

${ }^{1}$ University of South Australia, 101 Currie St, Adelaide, SA 5001, Australia

${ }^{2}$ James Cook University, 1 James Cook Drive, Townsville City, QLD 4811,

Australia
} 


\section{Background}

Indigenous Australians have the highest prevalence and incidence of diabetes in Australia [1] and also suffer high rates of preventable complications [2]. Many of these complications can be prevented with better primary care level management however access to culturally appropriate high quality diabetes care is not always evident, especially in remote settings where there is high turnover of health staff. Australian Indigenous adults with type 2 diabetes are on average 10 years younger, have poor glycemic control and lower levels of preventive service uptake compared to non-Indigenous adults with diabetes in a national sample [3]. As a consequence there are high rates of diabetes-related avoidable hospitalisations for people in remote settings [4]. Previous reports suggest that community health workers can contribute to improved diabetes care and outcomes in high risk and under-served patients in Australia [5,6] and elsewhere through more effective communication and culturally appropriate self-management support, although until recently, few studies use robust randomized controlled designs. Interventions with the strongest outcomes included "cultural tailoring of the intervention, community educators or lay people leading the intervention, oneon-one interventions with individualized assessment and reassessment, incorporating treatment algorithms, focusing on behavior-related tasks, providing feedback, and high-intensity interventions ( $>10$ contact times) delivered over a long duration ( $\geq 6$ months)" [7].

We report the results of a cluster randomised controlled trial, "Getting better at chronic care" which aimed to evaluate the impact of a case management approach by local community-based health workers supported by an Indigenous clinical outreach team in 12 primary care services in remote far north Queensland communities over an 18 month period from 2011 to 2013.

\section{Methods}

\section{Study design}

The study setting was 12 small remote communities (Indigenous population range 260-3,000) in far north Queensland where the majority of the population was Aboriginal or Torres Strait Islander, served by a single provider and where the health service had agreed to participate in the trial. Primary health care is provided by either a community-controlled service $(n=4)$ or the Queensland Government $(n=8)$. The distance to the nearest tertiary hospital is between one and 12 hours by road or air.

The unit of randomisation was the community health service which was allocated to either the health-worker led case management intervention or to a waitlist control group (where the intervention was provided after
18 months). Following patient recruitment and baseline data collection, the 12 services were randomly allocated (names out of a hat) to either the intervention $(n=6)$ or waitlist group $(n=6)$. The study was not blinded as the allocation arm was known following recruitment and baseline data collection and the study was designed as a pragmatic trial reflecting effectiveness in real world practice [8]. The data reported here were collected at two timepoints. Baseline data at the time of recruitment (2011) and follow-up data in 2013.

The study was approved by the Cairns and Hinterland Institutional Ethics Committee with support from the peak Aboriginal and Torres Strait Islander Health Councils. The trial is registered as a clinical trial, ANZCTR number 12610000812099.

\section{Study sample}

Patient eligibility criteria included having type 2 diabetes and at least one major comorbidity, age 18 or more, poor glycemic control $(\mathrm{HbA} 1 \mathrm{c}>=8.5 \%$ or $69 \mathrm{mmol} / \mathrm{mol})$, mentally competent and able to provide informed consent, and obtaining regular care from the identified health service. Exclusion criteria were major mental ill health (major psychosis or depression requiring inpatient treatment) or substance misuse, renal dialysis or end-stage renal disease, a cancer diagnosis or current pregnancy. Eligible patients were identified from their records by the health service staff, who then approached them to be in the trial. Enrolment occurred between December 2011 and July 2012. Baseline (2011) and follow-up (2013) interviews were conducted by Indigenous researchers in either plain English or Creole, depending on the preference of the patients.

The trial was powered to demonstrate a reduction in median HbA1c by $1.0 \%$ over 18 months compared to baseline, as the primary outcome measure. This estimate was based on a mean drop of $1.3 \%$ HbA1c over one year (from 9.9\% to 8.6\%, following initiation of intensive drug treatment in T2DM) reported by a large US Health Maintenance Organization [9]. A sample size of 49 in each group would have $90 \%$ power to detect a difference in mean HbA1c between the intervention and control group after 18 months of $1.0 \%$, assuming that the common standard deviation was $1.5 \%$ using a two group $t$-test with a 0.05 two-sided significance level. With 12 communities (6 intervention, 6 control), this would require 9 subjects per community. With adjustment for clustering, assuming a design effect of 1.2 derived from a similar study [10], the required number of subjects per community is 11 for the primary outcome, or 132 in total [11]. However, due to potential difficulty of maintaining subjects in these communities in the trial, high rates of mobility, and the potential for a more modest effect size in this group, we aimed to recruit 300 subjects. In the event, 327 patients were assessed by the health 
services as eligible and 213 (65\%) agreed to participate, providing written informed consent. Over the study period, 22 patients (10\%) were lost to follow-up: 6 died, 15 moved away from the community permanently and one withdrew. More patients in the intervention group than the waitlist group were lost to follow up (Figure 1).

\section{Intervention}

Each site allocated to the intervention arm recruited an Indigenous health worker resident in the community (selected by the health service) to work as part of the primary care team, and allocated a caseload of between 9 and 26 clients. The health workers with low caseloads worked part-time. All health workers at the commencement of the study received an intensive 3-week training in clinical aspects of diabetes and other chronic condition care, including how to support patients in self- management skills, advice on medications, routine foot care, nutrition, smoking cessation, follow up referrals to other providers, and scheduled tests. The roles of the health workers included helping patients make and keep appointments, understand their medications and nutrition and the effects of smoking and where appropriate, work with the family to help support the patient in selfmanagement. Home visits and out-of-clinic care were features of the trial, however visits were conducted according to the patients' preferences.

The curriculum included specific training and practice in:

- Rationale for the chronic care model and evidence-based management and treatment goals in diabetes, hypertension, COPD, renal disease and CHD.

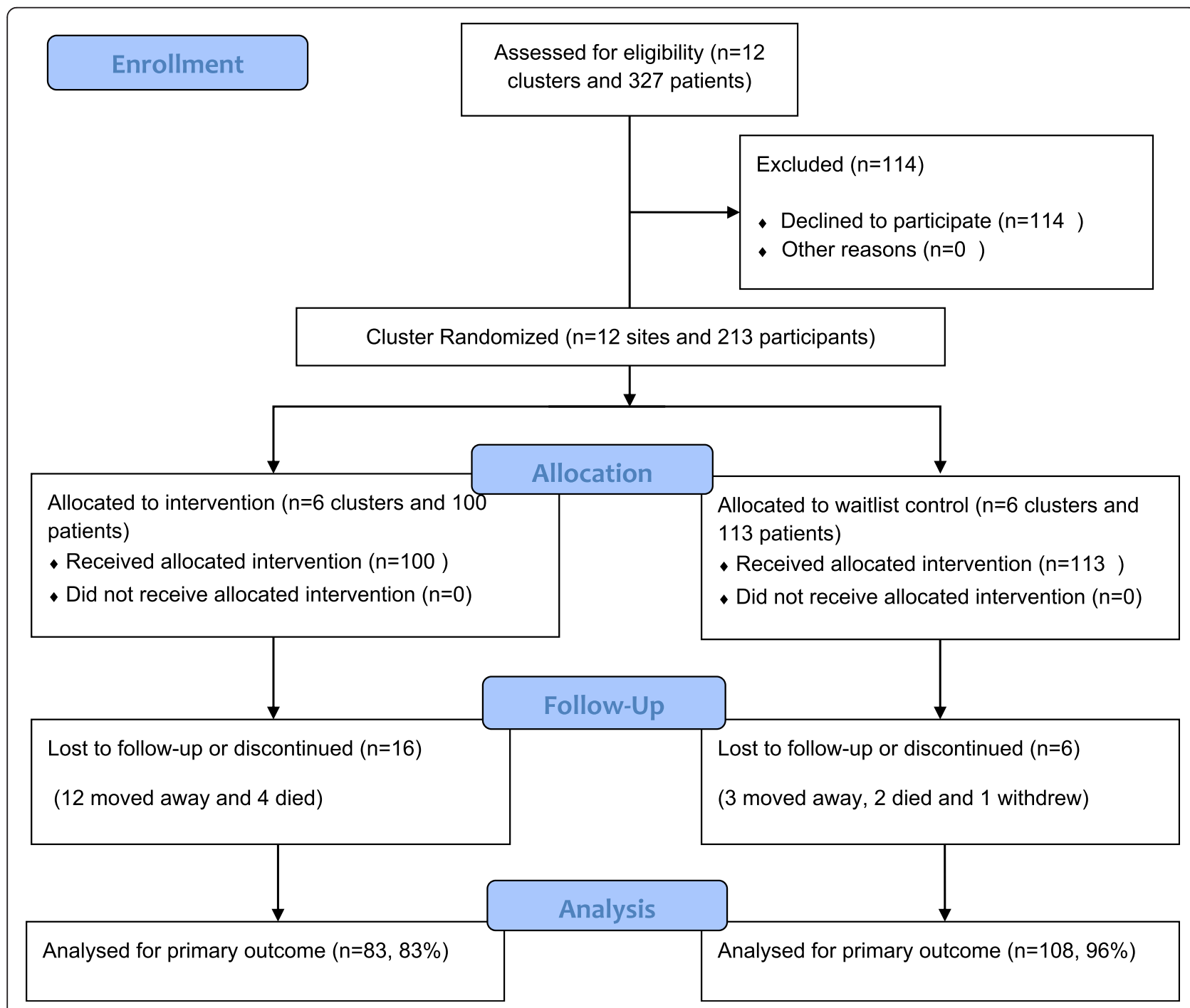

Figure 1 CONSORT flow diagram: getting better at chronic care cluster RCT. 
- "Hands-on" case management: regular client home visits, including basic diabetes care (scheduled clinical checks and blood tests, counselling and referral as per the clinical guidelines supported by the clinical team).

- Working in a primary care team, with clear roles and responsibilities of team members.

- Engaging with families and using local resources to support effective client self-management.

During the 18 month intervention period, the health workers attended two workshops where they underwent refresher training, including in Good Clinical Practice and reflective practice. During these sessions, they reported on their patients' progress and shared approaches to problem solving with the clinical support team and peers.

\section{Measures}

The primary outcome measure for this trial, glycemic control (HbA1c) was measured by Queensland Medical Laboratories using standard high-pressure liquid chromatography methods. Blood pressure, height, weight, serum fasting lipids (Cholesterol fractions and triglycerides) were abstracted from clinic files and electronic records. Taking insulin was defined as having any of long-acting, medium- or short-acting insulin. Albuminuria was defined as urinary ACR $>=3.4 \mathrm{mg} / \mathrm{mmol}$. Estimated glomerular filtration rate (eGFR) was calculated from serum creatinine using the CKD-EPI formula, where GFR $=141 \times \min (\mathrm{Scr} / \kappa, 1)^{\alpha} \times \max (\mathrm{Scr} / \kappa, 1)^{-1.209} \times$ $0.993^{\mathrm{Age}} \times 1.018$ [if female] $\times 1.159$ [if black], where Scr is serum creatinine $(\mathrm{mg} / \mathrm{dL}), \mathrm{K}$ is 0.7 for females and 0.9 for males, $\alpha$ is -0.329 for females and -0.411 for males, min indicates the minimum of $\mathrm{Scr} / \mathrm{K}$ or 1 , and max indicates the maximum of $\mathrm{Scr} / \mathrm{K}$ or 1 [12].

Test of Functional Health Literacy for Adults (TOFHLA) [11] was administered at study enrolment to all participants to gauge the patients' general understanding of health messages and procedures. In general, TOFHLA was scored highly in both groups, and it was concluded that they would not have major difficulty working with the diabetes care team.

Quality of Life was estimated using the Assessment of Quality of Life (AQoL) instrument, a multi-attribute utility instrument developed using Australian importance weights [13]. Socio-demographic data was by selfreport, including years of formal education, household income, employment, food insecurity ("Do you frequently not have enough money to buy food?"), current smoking and medication adherence.

Guideline recommended clinical checks, including General Practitioner Management Plans (GPMP) and specialist referrals [14], were abstracted from primary care records for the 18 months prior to the baseline and follow-up assessments. For patients with clinical indications, appropriate medication use (as indicated in the clinical guidelines) was recorded, including insulin, statins, ACEi or ARB drugs, vaccination.

Implementation Fidelity and "dose" was estimated through diaries kept by the IHWs which recorded time spent on specific intervention-related tasks versus other clinical work, as we anticipated that due to the small size of the communities and the limited number of health staff, that the trial workers would be called upon to perform regular acute clinical care to the general patient population.

\section{Statistical analysis}

Baseline demographic and clinical data were analysed for differences between intervention and waitlist patients using t-tests, Wilcoxon rank sum tests and chi-square tests as appropriate. At follow-up all analyses were on an intention to treat basis. The 22 people who had either died or were lost to follow up were included in the dataset; however, their 18 month follow-up results were left blank. Analysis was by Generalized Estimating Equations (GEE), using a Gaussian family and identity link function. The GEE regression models used Group, Time, and a Group $\times$ Time interaction term as predictor variables. The formal test of an intervention effect was whether or not the coefficient for the interaction term was statistically significant at the $5 \%$ level. An initial analysis using a mixed effects model showed that adjustment for clustering by community made little difference, based on a likelihood ratio test. That being the case, further models were not adjusted for clustering. There were a few cases where data was available for only one time point. Where there was missing data, a second analysis was performed used multiple imputation with 20 copies of the dataset and using a regression approach for imputation. Nonparametric tests (ranksum test) were used to examine HbA1c change by GPMP exposure between groups. A further analysis was done looking at the impact of the service model (Community controlled versus Government provided) on the likelihood of clinical change, independently of group allocation.

All analyses were done using Stata version 13.

\section{Results}

At baseline, there were no significant differences between allocation groups in age (mean age 47.9 years), sex ratio (62\% women), employment status, years of schooling, median household income, self-reported food insecurity, household size, median AQoL score on the mental health scale, smoking prevalence, HbA1c (10.7\%) and mean BMI (32.5). The intervention group scored lower on the health literacy test (Table 1). 
Table 1 Baseline socio-demographic characteristics of study participants (SD or \%)

\begin{tabular}{|c|c|c|c|c|}
\hline & Control $(95 \% \mathrm{Cl})$ & Intervention $(95 \% \mathrm{Cl})$ & All $(95 \% \mathrm{Cl})$ & p-value \\
\hline Number of participants & 113 & 100 & 213 & \\
\hline Mean age (years) & $47.8(46.2-49.5)$ & $47.9(45.8-50.0)$ & $47.9(46.6-49.2)$ & 0.948 \\
\hline Number (\%) women & $66.4(57.6-75.2)$ & $58.0(48.2-67.8)$ & $62.4(55.9-69.0)$ & 0.208 \\
\hline Unemployed (\%) & $52.2(42.9-61.5)$ & $40.0(30.3-49.7)$ & $46.5(39.7-53.2)$ & 0.204 \\
\hline Did not complete 12 years education (\%) & $61.9(52.9-71.0)$ & $73.0(64.2-81.8)$ & $67.1(60.8-73.5)$ & 0.344 \\
\hline Median annual (IQR) household income (\$) & $17420(12480-33800)$ & $20215(13585-31200)$ & $18200(13000-32500)$ & 0.598 \\
\hline "Not enough money for food" (\%) & $40.7(31.6-49.9)$ & $37.0(27.4-46.6)$ & $39.0(32.4-45.6)$ & 0.580 \\
\hline Median score (IQR) TOFLA & $90.0(81.1-94.1)$ & $80.6(64.9-89.0)$ & $86.1(71.5-92.1)$ & $<0.001$ \\
\hline No of people per household median (IQR) & $5(3-7)$ & $4(3-7)$ & $4(3-7)$ & 0.608 \\
\hline Median AQoL mental health score (IQR) $\max =1$ & $0.93(0.89-0.98)$ & $0.93(0.91-0.94)$ & $0.93(0.89-0.95)$ & 0.688 \\
\hline Current smoker (\%) & $37.6(28.4-46.8)$ & $40.2(30.3-50.1)$ & $38.8(32.1-45.5)$ & 0.231 \\
\hline Mean BMI $(\mathrm{kg} / \mathrm{m} 2)^{*}$ & $33.0(31.2-34.9)$ & $31.9(29.9-33.9)$ & $32.5(31.1-33.8)$ & 0.434 \\
\hline
\end{tabular}

*Missing data: $\mathrm{BMI} \mathrm{n}=113$.

At follow-up, 45.2\% (95\% CI, 34.5-56.0\%) of patients in the intervention group had a current GP Management Plan (GPMP) for diabetes compared to $35.5 \%$ (26.3-44.7) in the waitlist group (OR 1.23, 95\% CI, 0.72-2.22). There was no association between having a GPMP at follow-up and HbA1c change from baseline. This may be due to the fact that many GPMPs were done within 6 months of the follow-up data collection point, so the chance for the GPMP to have an immediate impact would be small. Further follow up may show a stronger relationship between having a GPMP and improved clinical indicators.
Other clinical care processes, including routine checks and specialist referrals, medications and self-reported smoking and medication adherence at baseline and follow-up, are summarised in Table 2. Intervention group patients were significantly more likely to have seen a dietician and dentist and slightly more likely to have seen a diabetes educator, be taking insulin and having influenza vaccination. Waitlist group patients showed greater self-reported adherence to prescribed medicines and were slightly more likely to have had an eye examination and be self-monitoring for glucose.

Table 2 Clinical care process at baseline and follow up (\%)

\begin{tabular}{|c|c|c|c|c|c|c|c|c|}
\hline & \multicolumn{4}{|c|}{ Baseline } & \multicolumn{4}{|c|}{ Endpoint (excluding 22 loss of follow up) } \\
\hline & \multicolumn{2}{|c|}{ Control $n=113$} & \multicolumn{2}{|c|}{ Intervention $\mathrm{n}=100$} & \multicolumn{2}{|c|}{ Control $n=107$} & \multicolumn{2}{|c|}{ Intervention $\mathrm{n}=\mathbf{8 4}$} \\
\hline & No & $\%(95 \% \mathrm{Cl})$ & No & $\%(95 \% \mathrm{Cl})$ & No & $\%(95 \% \mathrm{Cl})$ & No & $\%(95 \% \mathrm{Cl})$ \\
\hline GPMP for diabetes $\%$ & 19 & $22.2(13.5-30.9)$ & 28 & $40.3(29.2-51.4)$ & 24 & $29.6(19.5-39.7)$ & 27 & $40.3(28.4-52.2)$ \\
\hline Foot check\% & 50 & $44.2(35.0-53.5)$ & 31 & $31.0(21.8-40.2)$ & 38 & $35.5(26.3-44.7)$ & 26 & $31.0(20.9-41.0)$ \\
\hline Seen by DM educator \% & 46 & $40.7(31.6-49.9)$ & 52 & $52.0(42.1-61.9)$ & 41 & $38.3(29.0-47.6)$ & 44 & $52.4(41.6-63.2)$ \\
\hline Seen by dietician \% & 22 & $19.5(12.1-26.8)$ & 30 & $30.0(20.9-39.1)$ & 21 & $19.6(12.0-27.2)$ & 37 & $44.0(33.3-54.8)$ \\
\hline Dentist check \% & 20 & $17.7(10.6-24.8)$ & 13 & $13.0(6.3-19.7)$ & 9 & $8.4(3.1-13.7)$ & 15 & $17.9(9.6-26.5)$ \\
\hline ECG check\% & 37 & $32.7(24.0-41.5)$ & 42 & $42.0(32.2-51.8)$ & 34 & $43.9(34.4-53.4)$ & 35 & $40.5(29.8-51.1)$ \\
\hline Eye check \% & 54 & $47.8(38.5-57.1)$ & 42 & $42.0(32.2-51.8)$ & 56 & $52.3(42.8-61.9)$ & 37 & $44.0(33.3-54.8)$ \\
\hline Smoker \% & 38 & $34.5(25.6-43.5)$ & 34 & $35.1(25.5-44.7)$ & 33 & $31.2(22.4-40.4)$ & 34 & $41.5(30.7-52.2)$ \\
\hline Blood glucose self-monitor \% & 45 & $40.9(31.6-50.2)$ & 46 & $46.0(36.1-55.9)$ & 63 & $59.4(50.0-68.9)$ & 44 & $52.4(41.6-63.2)$ \\
\hline Taking insulin\% & 55 & $48.7(39.4-58.0)$ & 40 & $40.0(30.3-49.7)$ & 47 & $43.9(34.4-53.4)$ & 40 & $47.6(36.8-58.4)$ \\
\hline Dislipidemia \% & 83 & $73.5(65.2-81.7)$ & 84 & $84.0(76.7-91.3)$ & 91 & $85.0(78.2-91.9)$ & 76 & $90.5(84.1-96.8)$ \\
\hline Taking lipid lowering medicines\% & 91 & $81.3(73.9-88.6)$ & 77 & $77.0(68.7-85.3)$ & 87 & $82.1(74.7-89.5)$ & 62 & $73.8(64.3-83.3)$ \\
\hline Albuminuria and taking ACEi or ARB drugs & 46 & $88.5(79.6-97.3)$ & 47 & $88.7(80.0-97.4)$ & 58 & $82.9(73.9-91.8)$ & 51 & $89.5(81.4-97.6)$ \\
\hline Adherent to all medicines & 53 & $46.9(37.6-56.2)$ & 55 & $55.0(45.1-64.9)$ & 57 & $53.3(43.7-62.8)$ & 41 & $48.8(38.0-59.6)$ \\
\hline Had Fluvax & 50 & $44.2(35.0-53.5)$ & 66 & $66.0(56.6-75.4)$ & 51 & $47.7(38.1-57.2)$ & 50 & $59.5(48.9-70.2)$ \\
\hline
\end{tabular}

The proportion calculated on available records. Taking insulin including having either long-term, medium-term or short-term insulin. Albuminuria defined as $A C R>=3.4 \mathrm{mg} / \mathrm{mmol}$; taking lipid lowering medicines including statin, fibrate, and lipase inhibitors. 
Despite very high rates of dyslipidemia there was generally very low uptake of lipid lowering treatment in both groups, and the high smoking rates were unchanged. Appropriate management of albuminuria was high in both groups.

There was a significant decrease in HbA1c of $1 \%$ from baseline in the intervention group, from 10.8\% (95 $\mathrm{mmol} / \mathrm{mol})$ to $9.8 \%(84 \mathrm{mmol} / \mathrm{mol})$ compared to the waitlist group, which showed a less marked decrease of $0.2 \%$ from $10.6 \%$ (92 $\mathrm{mmol} / \mathrm{mol}$ ) to $10.3 \%(89 \mathrm{mmol} / \mathrm{mol})$, $(\mathrm{p}=0.018)$. More in the intervention group achieved at least a $0.5 \%$ interval reduction in HbA1c (67.5\%, 57.3-77.7), than in the waitlist group (48.6\%, 38.9-58.2). There were small improvements in both groups for total cholesterol, LDL cholesterol, cholesterol: HDL ratio, with slightly better results in the intervention group. Blood pressure and weight decreased in the waitlist group and increased slightly in the intervention group. None of these effects were statistically significant at the $5 \%$ level (Tables 3 and 4).

Implementation "dose" (time reported by the Health Workers spent on project-specific tasks) ranged from $43 \%$ to $78 \%$ over the intervention period. Thus we expected some diminution of effect in intervention sites.

The impact of Health Service Model (community controlled (CC) versus not CC) on the likelihood of a participant having a GPMP was explored independently of whether the trial site was in the intervention or the control group allocation. The percentage of GPMPs completed in sites with a community-controlled service was $71.0 \%$ compared to $23.5 \%$ among the non-CC sites $(\mathrm{OR}=3.0,95 \%$ confidence interval, 1.2-7.5 after adjustment for clustering) (Table 5). However there were no differences in clinical measures between $\mathrm{CC}$ and nonCC sites at follow-up.

\section{Discussion}

Type 2 diabetes and associated cardiovascular and renal disease contribute more than $60 \%$ to the $11-13$ year life expectancy gap experienced by Indigenous Australians [15]. We found that a health-worker led case management approach to care of high risk adults with type 2 diabetes in remote communities in Australia was effective in improving some diabetes care processes and glycemia over 18 months. This model has been demonstrated recently in other countries and similarly disadvantaged and high risk populations [16-19] using robust evaluations, and suggest our results are generalizable to other similar rural or remote settings. However the relatively short-term $(<2$ years) follow-up in these studies does not allow capture of the longer term benefits. Economic evaluation therefore relies on modelling rather than empirical estimates of costs and benefits, although most reports favour low-cost primary care level interventions [20].

Limitations to our study include lower than expected patient recruitment, small numbers in two of the intervention sites, a relatively high loss to follow up (10\%) which was higher in the intervention group and missing data for some of the secondary clinical endpoints. The latter was due to the pragmatic nature of the trial where clinical data was extracted from patient records. Loss to follow-up in the intervention group did not appear to be related to the trial itself, but to family circumstances, where a greater proportion moved out of the community. Other factors which potentially limited the implementation effectiveness of the study were major health

Table 3 Clinical measures at baseline and follow-up by group, absolute values

\begin{tabular}{|c|c|c|c|c|c|c|c|c|c|c|c|c|}
\hline \multirow[t]{3}{*}{ Pathology tests } & \multicolumn{6}{|c|}{ Baseline } & \multicolumn{6}{|c|}{ Endpoint (excluding 22 lost to follow up) } \\
\hline & \multicolumn{3}{|c|}{ Control $n=113$} & \multicolumn{3}{|c|}{ Intervention $\mathrm{n}=100$} & \multicolumn{3}{|c|}{ Control $n=107$} & \multicolumn{3}{|c|}{ Intervention $\mathrm{n}=84$} \\
\hline & No. & Mean & SD & No. & Mean & SD & No. & Mean & SD & No. & Mean & SD \\
\hline $\mathrm{HbA1c}$ & 113 & 10.6 & 1.87 & 99 & 10.8 & 2.0 & 105 & 10.3 & 2.2 & 84 & 9.8 & 2.3 \\
\hline s.creatinine & 97 & 77.5 & 43.8 & 88 & 77.7 & 39.7 & 102 & 92.0 & 85.0 & 83 & 107.4 & 138.6 \\
\hline eGFR* & 97 & 109.8 & 26.7 & 88 & 114.1 & 31.0 & 102 & 104.5 & 30.8 & 83 & 103.3 & 33.4 \\
\hline UACR & 78 & 63.9 & 138.9 & 73 & 71.3 & 169.4 & 96 & 102.8 & 233.9 & 79 & 92.1 & 167.9 \\
\hline Cholesterol & 87 & 4.6 & 1.3 & 81 & 4.5 & 1.3 & 100 & 4.7 & 1.3 & 79 & 4.4 & 1.4 \\
\hline Trig & 86 & 2.5 & 1.9 & 81 & 2.1 & 1.4 & 100 & 2.7 & 1.8 & 79 & 2.5 & 1.8 \\
\hline $\mathrm{HDL}$ & 72 & 1.1 & 0.6 & 79 & 0.9 & 0.2 & 99 & 0.9 & 0.2 & 78 & 0.9 & 0.2 \\
\hline LDL & 65 & 2.6 & 1.0 & 76 & 2.7 & 1.1 & 95 & 2.6 & 1.1 & 71 & 2.4 & 0.9 \\
\hline Chol-HDL ratio & 72 & 5.2 & 1.6 & 77 & 5.4 & 1.6 & 99 & 5.0 & 1.4 & 77 & 5.0 & 1.7 \\
\hline Weight & 89 & 91.4 & 19.3 & 87 & 89.7 & 22.6 & 92 & 87.4 & 18.6 & 81 & 91.0 & 23.1 \\
\hline BP systolic & 109 & 134.0 & 20.9 & 95 & 127.9 & 16.7 & 100 & 133.6 & 19.4 & 84 & 132.5 & 17.7 \\
\hline BP diastolic & 104 & 81.0 & 11.1 & 92 & 77.7 & 10.4 & 103 & 81.3 & 11.4 & 84 & 77.8 & 9.9 \\
\hline
\end{tabular}

*Calculated from serum creatinine using CKD-EPI formula. 
Table 4 Differences between baseline and follow-up by group

\begin{tabular}{|c|c|c|c|c|c|c|}
\hline Measure & Group & No. & Mean difference & Std. error of the difference & $95 \% \mathrm{Cl}$ mean lower & 95\% Cl mean upper \\
\hline \multirow[t]{2}{*}{$\mathrm{HbA1c}$} & Control & 105 & -0.2 & 0.2 & -0.7 & 0.2 \\
\hline & Intervention & 83 & -1.0 & 0.2 & -1.4 & -0.5 \\
\hline \multirow[t]{2}{*}{ S Creatinine } & Control & 89 & 18.9 & 6.9 & 5.2 & 32.6 \\
\hline & Intervention & 74 & 32.7 & 13.4 & 6.0 & 59.5 \\
\hline \multirow[t]{2}{*}{ eGFR* } & Control & 89 & -7.2 & 1.8 & -10.7 & -3.7 \\
\hline & Intervention & 74 & -10.3 & 3.1 & -16.5 & -4.1 \\
\hline \multirow[t]{2}{*}{ UACR } & Control & 71 & 41.2 & 25.3 & -9.3 & 91.8 \\
\hline & Intervention & 58 & 17.6 & 20.1 & -22.7 & 57.9 \\
\hline \multirow[t]{2}{*}{ Cholesterol } & Control & 64 & -0.3 & 0.2 & -0.6 & -0.02 \\
\hline & Intervention & 60 & -0.6 & 0.2 & -0.9 & -0.3 \\
\hline \multirow[t]{2}{*}{ Triglycerides } & Control & 79 & 0.1 & 0.2 & -0.4 & 0.5 \\
\hline & Intervention & 64 & 0.2 & 0.2 & -0.1 & 0.5 \\
\hline \multirow[t]{2}{*}{$\mathrm{HDL}$} & Control & 64 & 0.06 & 0.02 & 0.02 & 0.09 \\
\hline & Intervention & 62 & 0.06 & 0.02 & 0.02 & 0.1 \\
\hline \multirow[t]{2}{*}{ LDL } & Control & 56 & -0.1 & 0.1 & -0.3 & 0.2 \\
\hline & Intervention & 57 & -0.3 & 0.1 & -0.6 & -0.1 \\
\hline \multirow[t]{2}{*}{ Cholesterol-HDL ratio } & Control & 64 & -0.3 & 0.2 & -0.6 & -0.02 \\
\hline & Intervention & 60 & -0.6 & 0.2 & -0.9 & -0.3 \\
\hline \multirow[t]{2}{*}{ Weight } & Control & 72 & -1.5 & 0.6 & -2.7 & -0.3 \\
\hline & Intervention & 71 & -0.6 & 0.7 & -2.0 & 0.8 \\
\hline
\end{tabular}

*Calculated using CKD-EPI formula.

Values in this table calculated as Endpoint- Baseline.

Please note, this calculation is based on only those patients for whom data was collected at both time points excluding 22 loss of follow up. There are a few cases where data was available for only one time point.

system reform occurring in the Queensland government health services generally during the life of the trial, which limited the ability of the service to recruit and retain essential staff. These changes disproportionately affected 3 of the intervention sites. Process evaluation found that all six health workers experienced higher workloads as the services pressured them to undertake clinical work in addition to their study caseload. This tended to dilute the potential impact of their work on the care of study patients.

\section{Conclusions}

In summary, we have demonstrated a significant and favourable impact on some diabetes care processes and glycemic control of a community health worker-led model

Table 5 Presence of a GPMP at T3 by health service model, independent of trial group allocation

\begin{tabular}{|c|c|c|c|c|}
\hline \multirow[t]{2}{*}{ GPMP } & \multicolumn{2}{|c|}{$C C n=62$} & \multicolumn{2}{|c|}{ Not-CC $n=151$} \\
\hline & No. & $\%(95 \% \mathrm{Cl})$ & No. & $\%(95 \% \mathrm{Cl})$ \\
\hline No & 18 & $29.0(17.6-40.5)$ & 114 & $76.5(69.6-83.4)$ \\
\hline Yes & 44 & $71.0(59.5-82.4)$ & 35 & $23.5(16.6-30.4)$ \\
\hline
\end{tabular}

of diabetes care in high risk disadvantaged populations in remote Australia. A longer term evaluation may enable more complete capture of further benefits, including economic impact on the health service and patient-important outcomes. As the rising incidence of obesity, diabetes and associated complications rises globally, it is clear that the current health care workforce, especially in low- and middle-income countries will not be able to adequately manage new and established cases using current workforce models [21]. Community Health Workers in high income countries have been shown to contribute significantly to chronic disease management especially in hard-to-reach populations [22], although urban settings might offer different challenges [23] and may play a more important role in the primary care team as the diabetes epidemic increases in these groups.

\section{Abbreviations}

ACEi: Angiotensin converting enzyme inhibitor; ACR: Albumin:creatinine ratio; ARB: Angiotensin receptor blocker; ANZCTR: Australian and New Zealand Clinical Trials Register; AQoL: Assessment of Quality of Life; CC: Community controlled; CHW: Community health worker; COPD: Chronic obstructive pulmonary disease; CHD: Coronary heart disease; GEE: Generalized estimating equations; GPMP: General practitioner management plan; HbA1c: Haemoglobin A1c; HDL: High density lipoprotein cholesterol; T2DM: Type 2 diabetes mellitus; TOFHLA: Test of functional health literacy for adults. 


\section{Competing interests}

$\mathrm{RM}$ is supported by NHMRC and QH Practitioner Fellowship and Barbara Schmidt and Sean Taylor are supported in part by a Fellowship funded under the Australian Primary Health Care Research Institute. The authors declare that they have no competing interests.

\section{Authors' contributions}

RM is principal investigator, conceptualised the project and designed the outline of the training curriculum, led the community consultation and study implementation and the manuscript writing team. AE wrote the statistical plan and ML prepared the data tables and conducted the preliminary analysis. AE undertook further statistical analysis including data imputation. BS managed the trial, including contract management, staff recruitment, convening advisory groups and liaising with stakeholders in the field. ST, VO and CP conducted baseline and follow-up interviews and data management and cleaning. $\mathrm{VO}$ and $\mathrm{CP}$ convened the health worker training program and follow up training in the field. All authors contributed to the manuscript preparation and review. All authors read and approved the final manuscript.

\section{Acknowledgements}

This study was funded by NHMRC and the Queensland Government, Partnership Grant No. 570149, and the Centre for Research Excellence in Primary Health Care. . The authors thank Rohan Pratt and Deb van Gaans for data management, Alex Stipcewitz for refining the study protocols, Wendy Hoy, Ashim Sinha and Mark Wenitong for clinical advice and the health workers, past and present on the project for their dedication to improving patient care and attention to detail. Finally the participating health services for their support for the health workers, and Apunipima Cape York Health Council, Gurriny and Mulungu health services as the peak Indigenous Community Controlled services supporting the project.

Received: 26 August 2014 Accepted: 12 January 2015

Published online: 19 February 2015

\section{References}

1. McDermott RA, Li M, Campbell SK. Incidence of type 2 diabetes in two Indigenous Australian populations: a 6-year follow-up study. Med J Aust. 2010;192(10):562-5.

2. Australian Institute of Health and Welfare. The Health and Welfare of Australia's Aboriginal and Torres Strait Islander Peoples. Canberra: AlHW, 2008 AlHW Cat No IHW 21.

3. McDermott R, Tulip F, Schmidt B. Diabetes care in remote northern Australian Indigenous communities. Med J Aust. 2004;180(10):512-6.

4. Queensland Health. The health of Queenslanders 2012: advancing good health. Fourth report of the Chief Health Officer Queensland. Brisbane: Queensland Health; 2012

5. McDermott R, Schmidt B, Sinha A, Mills P. Improving diabetes care in the primary healthcare setting: a randomised cluster trial in remote Indigenous communities. Med J Aust. 2001;174:497-502.

6. Si D, Bailie RS, Togni SJ, D'Abbs PHN, Robinson GW. Aboriginal health workers and diabetes care in remote community health centres: a mixed method analysis. Med J Aust. 2006;185(1):40-5.

7. Glazier RH, Bajcar J, Kennie NR, Willson K. A systematic review of interventions to improve diabetes care in socially disadvantaged populations. Diabetes Care. 2006;29(7):1675-88.

8. Campbell MK, Piaggio G, Elbourne DR, Altman DG. Consort 2010 statement: extension to cluster randomised trials. BMJ. 2012;345:e5661.

9. Karter A, Moffet H, Liu J, Parker M, Ahmed A, Ferrara A, et al. Achieving good glycemic control: initiation of new antihyperglycemic therapies in patients with type 2 diabetes from the Kaiser Permanante Northern California Diabetes Registry. Am J Manag Care. 2005;11(4):262-70.

10. Littenberg B, Maclean D. Intra-cluster correlation coefficients in adults with diabetes in primary care practices: the Vermont Diabetes Information System field survey. BMC Med Res Methodol. 2006;6:20.

11. Parker RM, Baker DW, Williams MV JRN. The test of functional health literacy in adults: a new instrument for measuring patients' literacy skills. J Gen Intern Med. 1995;10(10):537-41.

12. Levey AS, Stevens LA, Schmid $\mathrm{CH}$, Zhang YL, Castro 3rd AF, Feldman HI, et al. CKD-EPI (Chronic Kidney Disease Epidemiology Collaboration) a new equation to estimate glomerular filtration rate. Ann Intern Med. 2009;150(9):604-12.
13. Richardson J, Atherton Day N, Peacock S, lezzi A. Measurement of the quality of Life for economic evaluation and the assessment of quality of life (AQoL) mark 2 instrument. Aust Econ Rev. 2004;37(1):62-88.

14. Diabetes Australia. Diabetes management in General Practice. Canberra: Diabetes Australia, 2011 July 2011. Report No.

15. Vos T, Barker B, Begg S, Stanley L, Lopez A. Burden of disease and injury in Aboriginal and Torres Strait islander Peoples: the Indigenous health gap. Int J Epidemiol. 2009:38:470-7.

16. DePue JD, Dunsiger S, Seiden AD, Blume J, Rosen RK, Goldstein MG, et al. Nurse-community health worker team improves diabetes care in American Samoa: results of a randomized controlled trial. Diabetes Care. 2013;36(7):1947-53.

17. Spencer MS, Rosland A-M, Kieffer EC, Sinco BR, Valerio M, Palmisano G, et al. Effectiveness of a community health worker intervention among African American and latino adults with type 2 diabetes: a randomized controlled trial. Am J Public Health. 2011;101(12):2253-60.

18. Prezio EA, Cheng D, Balasubramanian BA, Shuval K, Kendzor DE, Culica D. Community diabetes education (CoDE) for uninsured Mexican Americans: a randomized controlled trial of a culturally tailored diabetes education and management program led by a community health worker. Diabetes Res Clin Pract. 2013;100(1):19-28.

19. Mohamed $\mathrm{H}$, Al-Lenjawi $B$, Amuna $\mathrm{P}$, Zotor $\mathrm{F}$, Elmahdi $\mathrm{H}$. Culturally sensitive patient-centred educational programme for self-management of type 2 diabetes: a randomized controlled trial. Primary Care Diabetes. 2013;7(3):199-206

20. Brownson CA, Hoerger TJ, Fisher EB, Kilpatrick KE. Cost-effectiveness of diabetes self-management programs in community primary care settings. The Diabetes Educator. 2009:35(5):761-9.

21. Mills A. Health care systems in low- and middle-income countries. N Engl J Med. 2014;370:552-7.

22. Perry Henry B, Zullinger Rose MRM. Community health workers in low-, middle-, and high-income countries: an overview of their history, recent evolution, and current effectiveness. Annu Rev Public Health. 2014;35:22. 1-.3.

23. Palmas W, Findley SE, Mejia M, Batista M, Teresi J, Kong J, et al. Results of the Northern Manhattan Diabetes Community Outreach Project: a randomized trial studying a community health worker intervention to improve diabetes care in hispanic adults. Diabetes Care. 2014;37(4):963-9.

\section{Submit your next manuscript to BioMed Central and take full advantage of:}

- Convenient online submission

- Thorough peer review

- No space constraints or color figure charges

- Immediate publication on acceptance

- Inclusion in PubMed, CAS, Scopus and Google Scholar

- Research which is freely available for redistribution 